\title{
Shockwave intravascular lithotripsy for multiple undilatable in-stent restenosis
}

\author{
Matteo Perfetti ${ }^{1}$, Nino Cocco ${ }^{1}$, Francesco Radico ${ }^{2}$, Irene Pescetelli ${ }^{3}$, \\ Nicola Maddestra ${ }^{1}$, Marco Zimarino, ${ }^{1,2}$ \\ ${ }^{1}$ Interventional Cath Lab, ASL 2 Abruzzo, Chieti, Italy \\ ${ }^{2}$ Institute of Cardiology “G. d'Annunzio University”, Chieti, Italy \\ ${ }^{3}$ Cardiovascular Department, ASST Papa Giovanni XXIII, Bergamo, Italy
}

A 72-year-old patient was admitted for unstable angina; he had undergone previous percutaneous coronary intervention (PCI) with drug-eluting stents (DES) implantation on the right coronary artery (RCA) and left anterior descending artery (LAD) 7 years prior. In-stent restenosis (ISR) was documented on both sites. During PCI of RCA a non-compliant balloon ruptured (Suppl. Video 1), causing cardiac arrest. After cardiac resuscitation and adrenaline infusion the procedure was aborted. For the persistence of rest angina, a further attempt was planned with a plaque modification strategy.

Angiography and optical coherence tomography showed diffuse calcifications of the RCA (Fig. 1, upper panel), with a mid-RCA ISR caused by combined DES underexpansion and neo-atherosclerosis and a proximal de novo calcified severe stenosis. Shockwave intravascular lithotripsy (S-IVL; Shockwave Medical, Inc.) was delivered on both sites. A sirolimus eluting balloon (SEB) was then inflated inside the ISR and a DES deployed in the proximal segment, with a good final result (Fig. 1, mid panel).

In-stent restenosis of previous DES at mid-LAD (Fig. 1, lower panel, left side) was similarly due to both neo-atherosclerosis and underexpansion. Four S-IVL cycles followed by inflation of a SEB allowed a good final result (Fig. 1, lower panel, right side).

Shockwave intravascular lithotripsy produces mechanical waves propagating from the balloon (Suppl. Video 2) and such waves fracture calcifications without affecting soft tissues; it has been recently proposed for the treatment of both de novo calcified lesion and stent underexpansion. Here S-IVL was used for the treatment of ISR due to both neo-atherosclerosis and DES underexpansion. Further studies are needed to test the safety and efficacy of S-IVL in this subset.

Conflict of interest: None declared

Address for correspondence: Marco Zimarino, MD, PhD, Institute of Cardiology, “G. d'Annunzio” University - Chieti, C/o Ospedale SS. Annunziata, Via dei Vestini, 66013 Chieti, Italy, tel: +39-0871-41512, fax: +39-0871-402817, e-mail:m.zimarino@unich.it

Received: 18.04.2020 Accepted: 21.04.2020 


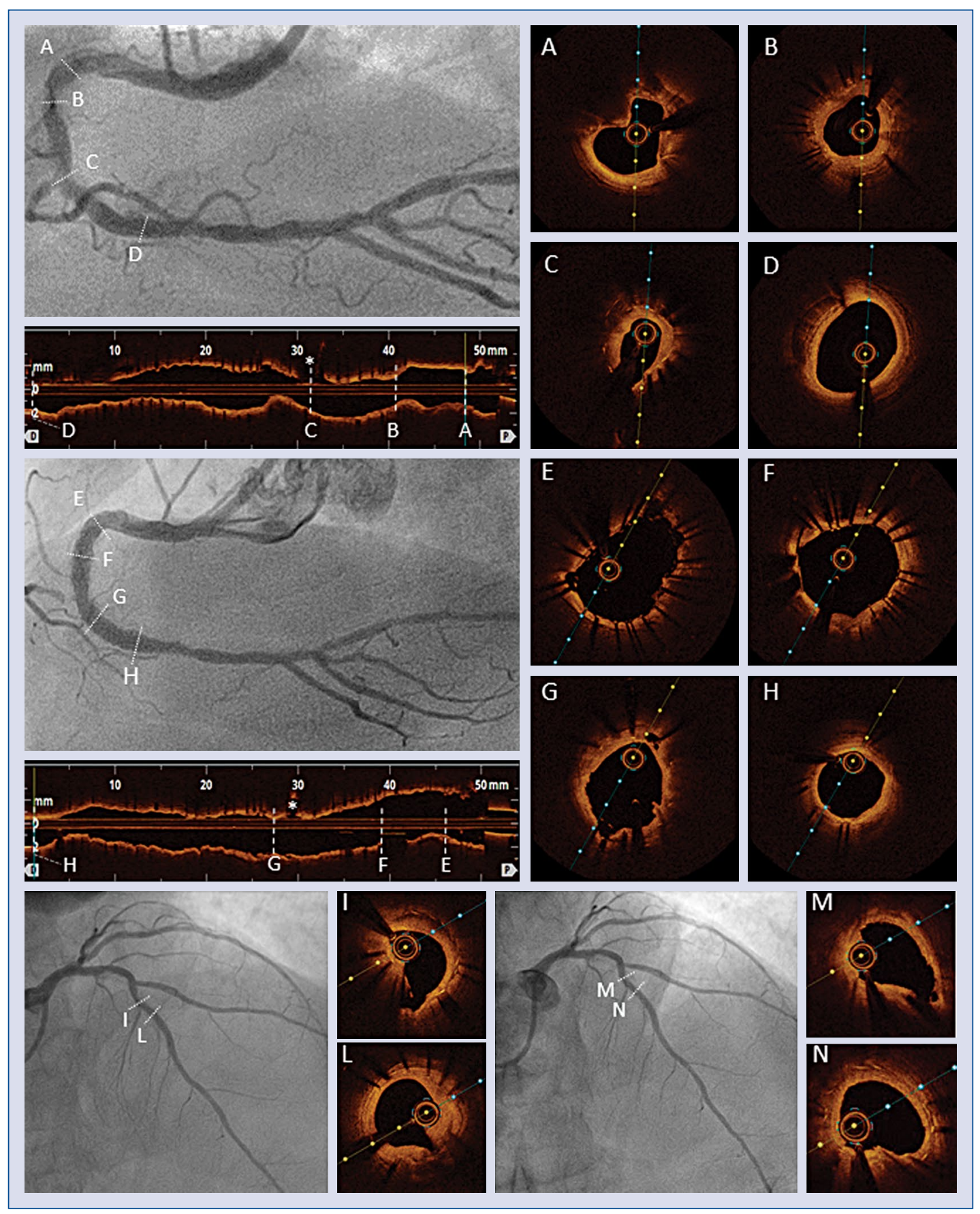

Figure 1. Upper panel: Baseline right coronary artery (RCA): angiography (upper left) of the RCA shows the position of 4 optical coherence tomography (OCT) cross-sections (upper right, A-D) reported on the right. A. Severely calcified eccentric de novo stenosis of the proximal segment; B. Concentric in-stent restenosis (ISR) with neointimal hyperplasia in the early midsegment. Stent underexpansion is detectable; C. In-stent restenosis/neo-atherosclerosis of the previously implanted two drug-eluting stents (DES) in overlap with underexpansion of the inner stent; D. Non-severe eccentric calcified plaque just below proximal to the DES edge; $\left({ }^{*}\right)$ Acute marginal branch; Mid panel: Angiography (left side) and OCT (right side) after 4 cycles of shockwave intravascular lithotripsy (S-IVL) with a $2.5 \times 20 \mathrm{~mm}$ balloon, subsequent noncompliant $3.0 \times 20 \mathrm{~mm}$ balloon and $3.5 \times 20 \mathrm{~mm}$ sirolimus eluting balloon (SEB) inflations inside the ISR and final new $4.0 \times 28 \mathrm{~mm}$ DES deployment between the proximal edge of the previous DES and de novo proximal RCA lesion; E, F. New DES has optimal deployment; G, H. Subsequent SEB dilatations achieved satisfactory plaque expansion inside the previously deployed DES; $\left({ }^{*}\right)$ Acute marginal branch; Lower panel: Angiography and OCT of baseline left anterior descending artery (left side) show a mid lesion, due to a combined mechanism of stent underexpansion and neo-atherosclerosis (I, L); after 4 cycles of S-IVL and inflation of a $3.5 \mathrm{~mm}$ SEB an optimal angiographic result is achieved (right side), with good DES expansion and adequate plaque fracture (M, N). 